\title{
Challenges in Treating Cancer Patients With Unstable Psychiatric Disorder
}

\author{
Val Bellman ${ }^{\mathrm{a}, \mathrm{h}}$, Nina Russell ${ }^{\mathrm{b}}$, Kartik Depala ${ }^{\mathrm{b}}$, Alexandra Dellenbaugh ${ }^{\mathrm{c}}$, Saral Desai ${ }^{\mathrm{d}}$, \\ Ramu Vadukapuram ${ }^{\mathrm{e}}$, Shweta Patel ${ }^{\mathrm{f}}$, Sushma Srinivas ${ }^{\mathrm{g}}$
}

\begin{abstract}
In this review, we first present a case of chronic myeloid leukemia with acute psychosis, and then we will discuss the incidence of cancer in patients with psychotic disorders, the manifestations of new-onset psychosis, and the prevalence of preexisting psychosis in cancer patients, coupled with their impact on the treatment, diagnosis, and prognosis of cancer. This was a case that presented with acute psychosis and was found to have an elevated white blood cell count upon admission to an inpatient psychiatric unit. He was diagnosed with chronic myeloid leukemia and successfully managed with imatinib/dasatinib therapy. Psychiatrically, he was stabilized on two long-acting injectable medications to help maintain adherence. We were able to eliminate his active psychotic symptoms and return him to normal functioning in affect and thinking, achieving sustained compliance with treatment. We identified multiple inconsistencies in screening for cancer of all types in these patients, masking of signs and symptoms that would typically clue physicians to the presence of cancers, underreporting of symptoms, and disparate access to healthcare resources in patients with mental disorders when compared to the general population. Treatment of cancer in these patients as compared to the general population has also been shown to be incongruent, which will be elaborated upon. Psychiatric interventions, as well as supportive measures, for treating patients who are facing challenges during active cancer treatment will be discussed.
\end{abstract}

Keywords: Leukemia; Psychosis; Mania; Cancer screening; Antipsychotics; Compliance

Manuscript submitted July 28, 2021, accepted August 17, 2021

Published online October 5, 2021

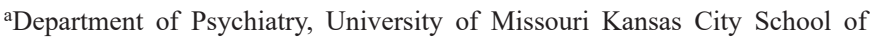
Medicine, 1000 E. 24th Street, Kansas City, MO 64108, USA

bUniversity of Missouri Kansas City School of Medicine, Kansas City, MO, USA

'University of Missouri-Kansas City, Kansas City, MO, USA

${ }^{\mathrm{d} D e p a r t m e n t}$ of Psychiatry, Brookdale University Hospital Medical Center, Brooklyn, NY, USA

eDepartment of Psychiatry, Icahn School of Medicine at Mount Sinai, New York, NY, USA

${ }_{\mathrm{f}}^{\mathrm{f}}$ Government Medical College, Surat, GJ, India

${ }^{g}$ A.J. Institute of Medical Sciences and Research Centre, NH66, Kuntikan, Mangalore, Karnataka, India

${ }^{\mathrm{h} C}$ Corresponding Author: Val Bellman, Department of Psychiatry, University of Missouri Kansas City School of Medicine, 1000 E. 24th Street, Kansas City, MO64108, USA. Email: vvzzw8@umkc.edu

doi: https://doi.org/10.14740/wjon1402

\section{Introduction}

Psychosis is broadly defined as impaired reality testing. Psychotic symptoms, such as hallucinations, delusions, paranoia, and agitation, may increase the risk of self-harm or risky behavior in patients, and can contribute to an inability to meet one's basic needs. Psychosis can be a symptom or complication of a multitude of psychiatric disorders, including thought disorders and mood disorders, and it can also be an acute manifestation of substance use or underlying medical conditions, such as cancer. This case report raises questions about whether patients burdened with serious mental illness are receiving appropriate cancer screenings and preventive care related to risk factors for cancer. It is equally important to continuously monitor, confront, and problem-solve patient actions that threaten to interfere with the continuation of chemotherapy, such as missing appointments, noncompliance, or abruptly leaving therapy.

\section{Case Presentation}

\section{Clinical manifestations}

The patient is a 39-year-old African American male, single, and unemployed, with a past medical history of psychotic disorders and recurrent manic episodes starting in 2018. This patient presented to the emergency department after making homicidal threats towards family members. Upon admission, the patient continued to act "erratically" with pressured speech and flight of ideas. He appeared to be disorganized, illogical, and constantly agitated. The patient was found to have an elevated white blood cell (WBC) count ranging between 41,000 to 92,000 , lactate dehydrogenase (LDH) of 767, and uric acid of 5.6 on admission to the behavioral health unit. Reportedly, he had been treated for psychosis four times in 2018 and 2019 before being admitted to our unit in September 2020. Each time, his WBC was elevated but considered to be reactive and not clinically significant.

\section{Course of treatment}

Upon admission to inpatient psychiatric unit in September 2020, the patient was diagnosed with chronic myeloid leu- 
kemia based on a detected P210 BCR-ABL1 fusion transcript and elevated multiple myeloid cell lines. Psychiatrically, the patient's presentation appeared to be consistent with schizoaffective disorder, bipolar type. He refused to have bone marrow biopsy done due to extreme and persistent distrust of others without a sufficient logical basis. Despite significant therapy to help understand his paranoid thinking, the patient did not display any insight into the problematic nature of his behavior and symptoms. Initially he was extremely resistant to treatment due to irrational persecutory beliefs (e.g., medication is poison, the physician is an enemy). He also could not initiate the proposed course of chemotherapy due to being violent as a defensive reaction to delusional or hallucinatory content.

\section{Chemotherapy and psychopharmacological management}

He was started on imatinib $400 \mathrm{mg}$ daily (September, 2020) but was lost to follow-up and had not been on treatment for 3 months due to psychiatric decompensation. He was restarted on imatinib and hydroxyurea in December, 2020 but developed prolonged pancytopenia in January, 2021. Subsequent discontinuation of hydroxyurea and transition to dasatinib were effective for improving thrombocytopenia as well as neutropenia.

Psychiatrically, he was stabilized on long-acting injectables (LAIs) paliperidone palmitate $(234 \mathrm{mg}$ ) and loxapine (100 $\mathrm{mg}$ ) orally (PO) twice a day (BID) in September, 2020. He was readmitted to the psychiatric hospital in December 2020, restarted on home medications, but was noted to be irritable and easily agitated, and thus mood stabilizer was considered. Patient was initially resistant to mood stabilizers and continued to demonstrate behaviors uncontrolled with gabapentin; thus, this was discontinued, and he was started on lithium. Patient was noted with increased sedation and slurring speech on lithium, and it was ultimately discontinued. By this point, the patient was overall showing improvement in his behaviors, so the psychiatry team opted not to trial a new antipsychotic. Given his non-compliance with oral medications, it was prudent to have him on two LAI medications as opposed to one LAI and oral agent. Patient's loxapine was discontinued, and he was started on $5 \mathrm{mg}$ of haloperidol PO BID with subsequent transition to haloperidol decanoate.

\section{Follow-up and outcomes}

Upon initial discharge in September 2020, he continued unhealthy behaviors (e.g., smoking, poor diet, use of mood-altering substances) despite a diagnosis of cancer and a physician's recommendation to alter these habits. Although he attended his oncology appointments, he would leave within 1 - 2 minutes of arriving. Three months later, he was readmitted to psychiatry, and a petition for a 21-day hold was granted. He was psychiatrically stabilized on two LAI medications. Psychiatry initially sought a petition for a 90-day hold, given difficulties in placement of the patient, but as he had shown significant improvement on the unit, the petition was dropped, and the patient was allowed to sign in voluntarily; he was later safely discharged to the community. The patient demonstrated meaningful improvement in health status and continued compliance with his outpatient treatment plan and visits to the oncology clinic within the next 90 days after discharge.

\section{Epidemiology of Psychotic Disorders Among Cancer Patients}

Numerous epidemiological studies have explored the prevalence of psychotic disorders and affective conditions in cancer patients. The findings of most of these studies appear to be paradoxical. Extensive population-based studies performed regarding the prevalence rate of schizophrenia and bipolar disorder have shown that prevalence has not changed drastically over the years. The prevalence rate for schizophrenia is between $0.3 \%$ and $1 \%$ of the general population [1]. Life expectancy is about $20 \%$ (15 - 20 years) shorter than those without the condition, especially in developed nations [1]. There has been a gradual rise in the prevalence rate over the past few decades, from 13.1 million cases in 1990 to 20.9 million cases in 2016 [2]. There is a $0.3-1.5 \%$ global prevalence rate for bipolar disorder, while in the past year, the US prevalence rate was about $2.9 \%$ among men and $2.8 \%$ among females [3].

The earliest hypothesis regarding the prevalence of cancer in schizophrenia developed in 1909 [1]. Since then, researchers have worked on the idea that patients with schizophrenia have a lower risk of developing cancer compared to the population without the condition. When reviewing historical studies exploring the prevalence of psychotic disorders in cancer patients, the results appear to be conflicting. A cross-sectional study of patients' electronic records by the Clalit Research Institute in Israel showed that women with schizophrenia spectrum disorders have higher prevalence rates of cancer than men with the same condition. A meta-analysis of standardized incidence ratios of cancer in patients with schizophrenia and their first-degree relatives was performed and compared with general population samples from the University of Queensland, Australia in 2008. It was found that the overall cancer incidence in patients with schizophrenia was not significantly increased (standardized incidence ratio $(\mathrm{SIR})=1.05$, confidence interval (CI): $0.95-1.15$ ). Lung cancer incidence was slightly increased (SIR $=1.31$, CI: $1.01-1.71$ ), but was reduced after adjusting for smoking prevalence. Breast cancer rates in female patients have significantly increased [4]. The higher incidence of breast cancer found in women with schizophrenia may likely, in part, be due to low parity, which is typical of women with psychiatric disorders. Some researchers have suggested that hyperprolactinemia, a side effect associated with neuroleptic drug therapy, might lead to an increased incidence of breast cancer [5].

Data from the Clalit Institute study also revealed that the prevalence rate of cancer of all types, and prostate cancer specifically, was lower in men with schizophrenia than in healthy controls [6]. The conclusion drawn by the Clalit Institute was that men with schizophrenia having apparently reduced cancer rates likely reflect under-diagnosis of certain cancer types, perhaps due to disparities in healthcare access [6]. 


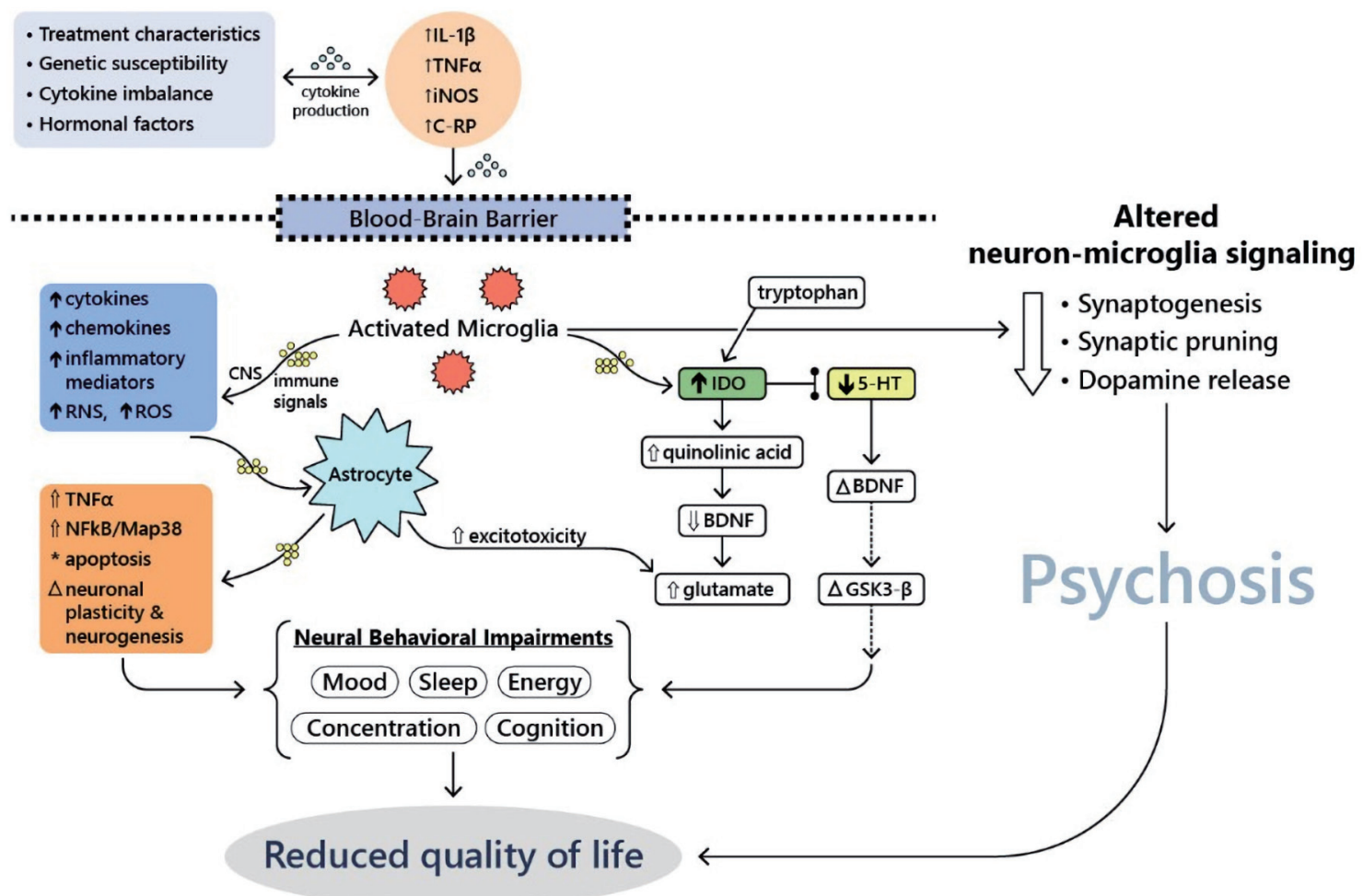

Figure 1. Molecular and cellular mechanisms underlying mood disturbances and psychosis in cancer patients (adapted from Watkins et al, 2014 [11]). 5-HT: serotonin; BDNF: brain-derived neurotrophic factor; CNS: central nervous system; C-RP, C-reactive protein; GSK3- $\beta$ : glycogen synthase kinase 3 beta; IDO: indoindoleamine 2,3-dioxygenase; IL-1 $\beta$ : interleukin-1 beta; iNOS: inducible nitric oxide synthase; Map38: p38 mitogen-activated protein kinase; NFkB: nuclear factor kappa-light-chain-enhancer of activated B cells; RNS: reactive nitrogen species; ROS: reactive oxygen species; TNFa: tumor necrosis factor alpha.

From an epidemiological perspective, the relationship between severe mental illness (SMI) and cancer prevalence may be skewed due to the generally shorter life expectancy associated with SMI. With the increased prevalence of tobacco use in this population coupled with the metabolic side effects of longterm antipsychotic use, death from cardiovascular causes often precludes potential cancers that they may have developed if they were to have longer life spans [7]. Although some cancers have a lower incidence in schizophrenia, as described below, it is important to note that patients with schizophrenia are still at an increased risk of metabolic and cardiovascular disease, and many of the risk factors for these disorders overlap with those for cancer.

In terms of biological links between SMI and cancer, multiple studies have shown an increased activity of natural killer cells in patients with schizophrenia, which may explain the lower rate of malignancy in patients with SMI, even with an increased rate of smoking [8]. However, the mortality rates of these patients due to cardiovascular causes remain high, and this can be a confounder, as these patients may die from these issues prior to cancer development.

There have also been six published incidence studies completed thus far that have analyzed the relative risk of cancer in patients with schizophrenia compared to the general population. These papers suggested that the tumor suppressor gene $p 53$ may play a role in the relatively decreased incidence of malignancy in patients with schizophrenia: $p 53$ may be overly active in these patients, thereby increasing levels of apoptosis at baseline and killing cells prior to becoming cancerous [9]. Recent evidence suggests that chemotherapy-induced changes may be associated with excessive neuroinflammatory response and subsequent behavioral decompensation [10]. Activated microglia may be involved in metabolism of inflammatory cytokines and neurotransmitters in patients with severe manic [11] or psychotic reactions [12]. Those changes may lead to long-term alteration of synaptic transmission, dopamine metabolism [12] and psychosis development [13]. Neuroinflammatory hypothesis may explain the therapeutic potential of various psychopharmacological agents in modulation of behavioral disturbances among cancer patients. For example, lithium can modulate adenylate cyclase-related GPCR signaling pathway and glycogen synthase kinase 3 beta (GSK-3 $\beta$ ) activity [14]. Figure 1 summarizes other potential molecular and cellular mechanisms underlying mood disturbances and psychosis in cancer patients.

There have also been multiple studies that noted increases in prolactin levels in patients with schizophrenia, likely due to the effects of prolonged antipsychotic use on the tuberoinfundibular system, and a concurrent increase in breast cancer risk in patients with SMI [15]. Overall, the data on the association between SMI and malignancy are limited, and at times, conflicting, as detailed above. This study provides an opportunity 
to elucidate the relationship between SMI and the prevalence of cancer going forward, and can serve as a basis to examine the discrepancies in data, as it provides a clear case of hematological malignancy developing in the setting of SMI.

\section{Manifestation of Psychosis and Mania in Can- cer Patients}

Psychosis is a symptom complex characterized by hallucinations, delusions, cognitive problems, and disorganized speech or behavior [16]. Medical disorders may present with psychosis first, necessitating a methodical approach to diagnosis to determine the reason. Thus, an oncologic origin, such as a steroid-producing tumor, a space-occupying brain lesion, or a paraneoplastic etiology, should be suspected if psychosis develops slowly [17]. SMI is described as schizophrenia or bipolar disorder that requires long-term therapy and results in disability or dysfunction [18]. Patients with SMI, such as schizophrenia, major depressive disorder, schizoaffective disorder, and bipolar disorder, have been shown to have shortened life expectancy by at least 13 - 30 years [19].

Cardiovascular disease and cancer are the primary causes of death in this population, implying that prompt and adequate preventive measurements could help reduce premature death [20]. Patients with SMI, particularly schizophrenia, are less likely to receive adequate cancer treatment, such as chemotherapy, radiation therapy, or surgery [21, 22]. Weinstein et al (2016) stated the key recommendations for cancer screening in this vulnerable population, such as providing a community health professional or peer counselor to assist the patient in navigating the screening process and making informed decisions and increasing mental health service providers' awareness of cancer screening [23]. By communicating effectively, providing routine emotional support, screening for psychiatric disorders, prescribing anxiolytic and antidepressant medications appropriately, referring patients to support groups, collaborating with mental health professionals, and dealing with end-of-life issues, oncologists can help patients with advanced cancer experience less psychological distress [24]. Discrepancies in cancer screening among patients with psychotic disorders surely contribute to the higher mortality rates mentioned previously.

When looking into the difficulties in diagnosing and treating cancer patients with psychotic or mood disorders, contrasting new onset psychosis and/or mania in cancer patients with exacerbation of psychosis or mood disorder is warranted. The most commonly reported new-onset psychiatric illnesses in cancer patients are adjustment disorders, anxiety, and depression related to developing the disease. Moreover, affective conditions have been proven to precipitate acute psychotic episodes in cancer patients. Difficulty arises in diagnosing newonset psychosis or mania in cancer patients because cancer, depending on type and location, is known to cause delirium. Delirium may present with psychosis but is typically precipitated by non-psychological stressors, and does not imply underlying mental illness. Delirium may be precipitated by dehydration, polypharmacy, infection, immobility (including restraint use), malnutrition, and the use of bladder catheters [25]. It is a typically reversible condition. However, in two separate studies, delirium was associated with increased mortality in cancer patients $[25,26]$. This adds further difficulty to the diagnosis of new-onset mental illness versus psychosis in cancer patients. Excluding delirium-induced psychosis, there is limited information available discussing new-onset psychotic disorders in cancer patients.

New-onset psychiatric illnesses have been associated with increased mortality in patients with cancer. A Swedish cohort of 1,615 patients who underwent surgery for esophageal cancer and had no prior psychiatric history were followed. It was found that the mortality rate of all causes was greater among patients who developed psychiatric disorders during or after treatment for their cancer [27]. A case report published by Bernad et al in 2013 examined the case of a 62-year-old man who developed anhedonia, paranoid delusions, insomnia, and irritability after treatment of prostate adenocarcinoma with external beam radiation and triptorelin (gonadotropin-releasing hormone agonist) therapy [28]. After his treatment ended and his psychosis resolved, it was concluded that his psychosis was likely due to androgen deprivation therapy used in the treatment of cancer, and he remained stable on aripiprazole $2 \mathrm{mg}$ at the 2-year follow-up [28]. Another case report published in the Journal of Pediatric Hematology-Oncology in 2011 analyzed a 16-year-old male patient who developed delusions, insomnia, and grandiosity following the diagnosis and initial treatment of a non-seminomatous germ cell tumor. He was initially treated with bleomycin, etoposide, and cisplatin (BEP) chemotherapy, along with dexamethasone, for the first 7 days of treatment. His psychiatric symptoms developed on day 14 of treatment, and dexamethasone was discontinued. The patient did not worsen following this, but he was stabilized on risperidone. It was concluded that his psychosis was most likely secondary to stress related to cancer diagnosis and treatment, history of traumatic brain injury (TBI) at age 5, and corticosteroid administration [29]. The patients examined in both of these case reports recovered from their respective cancers as expected and maintained psychiatric stability on antipsychotics.

Pre-existing psychotic disorders have been known to be exacerbated in patients with cancer. A cancer diagnosis can cause or exacerbate anxiety and depression in patients who are already afflicted with mental illness [30]. Patients with mental illness have higher mortality rates from cancer.

\section{Screening for Cancer in Psychiatric Patients}

Our patient had at least two episodes of elevated WBC count associated with exacerbation of affective and psychotic symptoms. The complete relationship between the immune system, psychosis, and mood symptom severity is not fully known; however, clinical studies have found a correlation between WBC, mood symptoms and psychosis. Two studies measured WBC with bipolar mood symptom severity and depression symptoms to assess whether cell count had any effect. For one trial, among the 482 participants, for each $1.0 \times 10^{9} / \mathrm{L}$ WBC deviation, the overall bipolar mood symptoms increased great- 
ly for men but not for women. The other trial showed that a higher deviation in median WBC showed an increasing trend in the Montgomery-Aasberg Depression Rating Scale scores among men but not women [31]. Another study analyzed 68 patients with schizophrenia during psychomotor excitation and then followed up about 1 month later during a medicated recovery phase. During psychomotor excitation, abnormal lab values were prevalent. Of these lab values, the most frequent ones seen in $\geq 35 \%$ of patients were low serum potassium, high levels of uric acid, fasting blood glucose, lactate dehydrogenase, and WBC count [32]. It is believed that there is an inflammatory mechanism that triggers increases in WBC in patients with mood and psychotic symptoms. Leukocyte counts in the serum samples of 28 children with new-onset psychosis showed a significant increase in absolute monocytes and lymphocytes in comparison to children without psychosis. Although these trials have shown statistically significant correlations between the WBC count and mood/psychotic symptoms there are several challenges. Only a small number of individuals are studied, as it is not only difficult to enroll patients with mood disorders; it is also challenging to follow up with them [33]. Screening patients with these symptoms can also be very challenging.

In reviewing previous studies on cancer screening in patients with mental disorders, a few in particular stand out. In a meta-analysis of Howard et al, multiple studies found no difference in screening rates when the rates of cancer screening of patients with mental disorders (including schizophrenia and manic-depressive psychosis) were compared with those of the general population [5]. Others, however, found that there were lower rates of cancer screening in patients with mental disorders when compared to a control group. The reason for the clear conflict in these findings is difficult to pinpoint. Within the meta-analysis, it was noted that most of the studies that found no difference in screening rates employed fewer participants with psychotic disorders compared to other mental illnesses [5]. Druss et al found that screening uptake was lower for patients with psychosis than for the general population, and that a coexisting substance abuse disorder reduced uptake even further [34]. Possible reasons suggested for this phenomenon are low income, lack of transportation, lack of familiar healthcare providers, and lack of communication with healthcare providers.

Patients with schizophrenia spectrum disorders often lack access to effective cancer screening or medical attention [6]. Patients with psychotic disorders tend to resist cancer screening. Moreover, patients lost to follow-up or shifting away from treatment could also be responsible for variations in the prevalence result, especially among men. A further explanation for this diverse prevalence, especially relating to the finding that women have an increased rate of cancer, has been linked to different studies identifying breast, cervical, or uterine cancer as high-risk cancer types [35]. Another study focused on identifying mental disorders before the diagnosis of breast cancer showed an increase in the prevalence of mental disorders in one-fourth of Medicare-enrolled women [36]. However, Kahan et al (2018) showed no statistically significant association between breast cancer and bipolar disorder in women [37]. In another study, most of the excess cancer risk was found in males with bipolar disorder compared to those with schizophrenia [38]. The overall trend of a generally lower incidence of cancer in schizophrenic men might be accurate, especially when we consider some forms of cancer that occur in men but usually remain undetectable for years, such as prostate cancer. Lastly, patients with psychotic disorders tend to smoke twice as often as the normal population. As a result, they are expected to have an increased risk of lung cancer. However, most epidemiological studies have not found a direct link to explain the relationship between the current prevalence rate of schizophrenic individuals and the risk of lung cancer [39]. The presence of mental illness prior to the diagnosis of cancer creates both diagnostic and treatment challenges. Overall, the literature discussed suggests that individuals with SMIs are less likely than other groups to receive screening for cancers of all types.

\section{Management of Cancer in Patients With Severe Psychotic and Bipolar Disorders}

In patients with severe psychotic and bipolar disorders, the management of cancer presents difficulties of its own. Chronic somatic comorbidities in schizophrenia or bipolar disorder result in excessive mortality, as stated above. These somatic comorbidities are often under-treated and under-detected [40]. Higher mortality rates from cancer in patients with mental disorders could be multifactorial, including the initial presentation of their cancer not receiving appropriate attention to challenges in the treatment of psychiatric patients [41].

It has been posited that psychiatric patients are less likely to report or seek treatment for telltale symptoms of cancer. One such factor underlying this phenomenon could be a difference in pain threshold. Atik et al (2007) proposed that patients with schizophrenia have a higher pain threshold when compared to the general population, which may make them resistant to seeking treatment for pain due to underlying cancer [42]. However, this is likely inaccurate for patients with bipolar disorder, as it has been associated with increased pain sensitivity [42]. People with medical conditions and co-existing mental illnesses are less likely to be treated for general medical conditions than people with medical conditions and no existing mental illnesses [43].

Discussion of nonmedical barriers to the management of cancer in patients with psychotic disorders is warranted. Patients presenting with acute psychosis may be difficult to assess physically or mentally. This may be due in part to largely inadequate follow-up found in this population, or due to the behavior typical of acutely psychotic patients. Patients in a psychotic state may be non-communicative, interruptive, paranoid, or even combative, especially with unfamiliar caregivers. Even after diagnosis, psychiatric patients, particularly those with schizophrenia, may still have difficulty understanding their diagnoses or the necessary treatments [30]. Inagaki et al also pointed to the distinct possibility of exacerbating a patient's symptoms of schizophrenia when informing them of their cancer diagnosis [30]. This is a unique barrier to treatment specific to cancer patients with SMI, and there has been little research 
Table 1. Commonly Used Antipsychotics in Oncology Settings

\begin{tabular}{|c|c|c|c|c|}
\hline Antipsychotic agent & Starting dose (mg) & Scheduling & Maximum daily dose (mg) & References \\
\hline Quetiapine & $25-50 \mathrm{mg}$ PO & Q12 - 24h & $800 \mathrm{mg}$ & $\begin{array}{l}\text { Sakamoto et al, } 2020 \text { [51]; } \\
\text { Shinno et al, } 2007 \text { [52]; Verwimp- } \\
\text { Hoeks et al, } 2012 \text { [53] }\end{array}$ \\
\hline Olanzapine (Zydis) & $2.5-10 \mathrm{mg}$ PO & Q12 - 24h & $20 \mathrm{mg}$ & $\begin{array}{l}\text { Sanomachi et al, } 2017 \text { [54]; Bitter et } \\
\text { al, } 2004 \text { [55]; Breibart et al, } 2002 \text { [56] }\end{array}$ \\
\hline Risperidone (M-Tab) & $0.25-2 \mathrm{mg}$ PO & Q12h & $6 \mathrm{mg}$ & $\begin{array}{l}\text { Kishi et al, } 2012[57] ; \\
\text { Reutfors et al, } 2017 \text { [58] }\end{array}$ \\
\hline Haloperidol & $0.25-1 \mathrm{mg}$ PO or IV & $\mathrm{Q} 2-12 \mathrm{~h} \mathrm{PO} / \mathrm{IV} / \mathrm{SQ}$ & $20 \mathrm{mg}$ & $\begin{array}{l}\text { Radha Krishna et al, } 2012 \text { [62]; Hui et } \\
\text { al, } 2017 \text { [63]; Wang et al, } 2012 \text { [64] }\end{array}$ \\
\hline Loxapine & $\begin{array}{l}10-50 \mathrm{mg} \text { PO. The } \\
\text { usual therapeutic range } \\
\text { is } 60 \text { - } 100 \mathrm{mg} \text { daily. }\end{array}$ & Q12h & $250 \mathrm{mg}$ & $\begin{array}{l}\text { Riddle et al, } 2017 \text { [65]; Schmiedl et } \\
\text { al, } 2019 \text { [66]; Rahman et al, } 2014 \text { [67] }\end{array}$ \\
\hline
\end{tabular}

PO: orally; IM: intramuscularly; IV: intravenous; q12h: every 12 hours.

on the methods of overcoming this barrier. These patients have also been shown to have lower adherence to treatment plans and are more likely to miss follow-up appointments. Kisely et al studied the reason that psychiatric patients had a $30 \%$ higher cancer mortality rate than the general population [41]. After performing a population-based record linkage analysis, they concluded that although cancer incidence is not higher in psychiatric patients than in the general population, these patients are more likely to have metastases at diagnosis. It was hypothesized that cancer is more likely to be unrecognized in psychiatric patients than in the general population, and that they were less likely to receive equitable primary care. This is supported by the finding that psychiatric patients were less likely to receive weight measurements, blood pressure measurements, and treatment for hyperlipidemia, particularly in the USA, Canada, UK, and Australia in 2000 [44]. They also found that psychiatric patients are less likely to receive specialized interventions, receive less chemotherapy, and are less likely to receive surgery after cancer diagnosis. Psychiatric patients are typically excluded from drug trials [41].

Competency to consent to treatment and decision-making capacity is also a consideration when it comes to incongruence in the treatment of cancer patients with psychotic disorders. Patients with psychotic disorders, such as schizophrenia, typically have a shorter lifespan when compared to the general population. This reveals a disparity regarding access to quality end-of-life care for these patients. Another barrier to treatment for patients with psychotic disorders is the phenomenon of diagnostic overshadowing by their treating physician. It is not uncommon for physicians to discount or pay less attention to the symptoms reported by their patients with psychotic disorders, and oftentimes, the physician will simply attribute these symptoms to the patient's mental illness. Symptoms of mental illness may therefore obscure the physician to the symptoms of cancer (or other illnesses) [45]. With this background, a clearer picture begins to form about inequality in care for psychiatric patients with a diagnosis of cancer.

\section{Role of Antipsychotic Medications}

Antipsychotic medications may have a role in cancer incidence in patients with mental disorders on the psychotic spectrum. For instance, as discussed earlier, women with schizophrenia have a higher incidence of breast cancer. It is believed that hyperprolactinemia-inducing antipsychotic medications can further progress pre-existing precancerous breast lesions [46]. However, many studies have shown that these medications do not increase the risk of breast cancer [47]. Some studies have even shown the anti-cancer properties of some drugs, such as aripiprazole [48]. Many antipsychotic drugs can inhibit cellular proliferation, induce apoptosis, inhibit migration or metastasis, cause cell cycle arrest, or alter the viability of cancer cells in vivo and in vitro. Thus, they may be used to treat various cancers at higher dosages $[49,50]$. Some data also show that these drugs can be used to augment chemotherapy and treat the side effects of chemotherapy [50]. Table 1 [51-69] summarizes the most commonly used antipsychotics in oncology settings.

Psychosis can be a pre-existing condition due to underlying mental illness or can be brought on by cancer or its treatment, and psychotic symptoms, including delusions, hallucinations, and disorganized behaviors/thoughts can be addressed by antipsychotic medications [70]. The selection of drugs depends on the patient's symptoms and the properties of the drug in question [71]. Haloperidol is the first-line drug for psychosis in cancer patients, followed by risperidone and low-dose quetiapine in general hospital settings [72]. 




Figure 2. Potential analgetic properties of certain antipsychotic agents.

Antipsychotic medications are known to have high rates of adverse effects. Both typical and atypical antipsychotics can cause electrocardiogram (ECG) abnormalities, which put them at a higher arrhythmia risk, and they increase the risk of coronary events, both of which lead to chances of sudden cardiac death $[73,74]$. Clozapine is specifically known to cause cardiotoxicity and myocarditis, which should be taken into consideration in patients on chemotherapy [75]. Second-generation antipsychotics cause metabolic side effects, such as weight gain, dyslipidemia, hyperglycemia, and insulin resistance, all of which can cause mortality and are risk factors for cardiovascular disease [76]. Antipsychotic use can cause bleeding, venous thromboembolism, and platelet function impairment, which increases the chances of stroke, thromboembolism, and cardiovascular events [77]. These side effects may increase the morbidity and mortality of cancer patients being treated for psychiatric illnesses. First, these symptoms could further mask the signs and symptoms of cancer and become barriers to diagnosis and screening. Second, these side effects may contribute to mortality when combined with certain types of cancer afflicting a patient.

Atypical antipsychotics can be utilized for various types of chronic pain, but an intriguing opportunity for use in treating cancer pain is being researched. Oftentimes, cancer pain is chronic, as most people battle cancer for longer than 3 months at a time. One study showed that around $59 \%$ of patients experienced pain during cancer treatment. About $64 \%$ of these patients reported advanced cancer pain. Even for patients with curative treatment, 33\% still claim to experience pain [78]. Regarding already established guidelines, the World Health Organization (WHO) cancer pain management guidelines suggest using non-opioid analgesics when initiating therapy for a patient. This is due to multiple factors: opioids are not only highly addictive but also have many side effects. A small study that assessed the benefit of atypical antipsychotics for the pain of eight cancer patients who were already titrated on opioids found that the drugs, unfortunately, did not control the pain. Using olanzapine as the drug of choice, the patients reported a significant reduction in their daily pain scores, and it was also noted that their daily opioid use decreased a great amount as well. The patients also had cognitive disorders and showed positive improvements in cognitive impairment and anxiety [79]. Further, when studied on mouse models, quetiapine showed analgesic effects on cancer-induced bone pain [80]. There is still much more to learn regarding the treatment of cancer pain with atypical antipsychotics. Currently, the theory is mainly supported through animal models and small cases studies. Nonetheless, antipsychotics have generally shown efficacy in pain management across the board and are an innovative approach to treating chronic pain in cancer patients with concomitant psychosis. Figure 2 summarizes analgetic properties of certain antipsychotics.

\section{Chemotherapy-Induced Mania: Should We Use Mood Stabilizers?}

Several chemotherapy regimens have been linked to secondary mania and agitation in patients without a previous psychiatric history. Specifically, it was suggested that our patient partially relapsed after the cycle of imatinib, as this coincided with the onset of psychomotor agitation and several episodes of behavioral dysregulation. After readjustment of antipsychotic therapy, symptoms subsided within 15 days. Table 2 [17, 81-89] summarizes chemotherapeutic agents associated with similar presentations.

Lithium, a commonly used mood stabilizer, has shown many antineoplastic properties that may contribute to reducing cancer incidence in psychiatric patients [90]. Lithium can inhibit the growth of cancer cells and inhibit cancer cell invasion and metastasis [91, 92]. Valproate, another mood stabilizer, is a histone deacetylase inhibitor that exhibits antineoplastic activities both in vivo and in vitro [93]. Although the exact mechanism of action is not understood, both lithium and valproic acid may be used to augment the effect of chemotherapy agents in cancer patients $[93,94]$. However, cancer patients on mood stabilizers are at a higher risk of developing serious adverse effects. For example, lithium levels can fluctuate greatly in cancer patients taking nephrotoxic chemotherapeutic agents. Similarly, carbamazepine may cause serious bone marrow suppression when administered alongside chemotherapeutic agents, causing bone marrow suppression [95]. 
Table 2. Chemotherapy-Induced Affective and Behavioral Disturbances

\begin{tabular}{|c|c|c|c|}
\hline Agent & Malignancy & Presentation & References \\
\hline 5-fluorouracil & Colorectal cancer & Acute manic episode & Ha et al, 2011 [81] \\
\hline Methotrexate & Acute lymphocytic leukemia (ALL) & Acute manic episode & $\begin{array}{l}\text { Hariram et al, } \\
2013 \text { [82] }\end{array}$ \\
\hline Cisplatin & Tonsillar cancer & Mania and poor impulse control & Barlinn et al, 2015 [83] \\
\hline Docetaxel and cisplatin & Non-small cell lung cancer & Late-onset bipolar disorder & DeKay et al, 2011 [84] \\
\hline Capecitabine and oxaliplatin & Colorectal cancer & Acute manic episode & Garg et al, 2018 [17] \\
\hline Dexamethasone & Non-small cell lung cancer & Steroid-induced mania & Warren et al, 2019 [86] \\
\hline Lenalidomide, bortezomib, dexamethasone & Multiple myeloma & Mania and hyperactive delirium & Pavlova et al, 2020 [87] \\
\hline Dasatinib & Chronic myeloid leukemia & Depression with agitation & Sami et al, 2014 [88] \\
\hline
\end{tabular}

\section{Psychotherapeutic Treatment Modalities}

Cancer is a stressful life event that may cause psychological stress and new-onset depression or anxiety, but cancer or its treatment can also exacerbate symptoms of preexisting schizophrenia and bipolar disorder [70]. Psychotherapy has been proven to help reduce negative thoughts, help patients develop adaptive defenses, and help family members enhance support for the patient at home $[95,96]$. Psychotherapy may not cure the symptoms of schizophrenia or bipolar disorder in cancer patients, but it can be moderately helpful in treating some depressive symptoms [97, 98]. Acceptance and commitment therapy, a type of therapy involving meaning and mindfulness developed by Steven Hayes, can be used for pre-existing psychiatric conditions in cancer patients [99]. Cancer support groups or group therapies can be used to keep patients motivated, help them relieve anxiety, and educate them [100].

A qualitative study performed in Australia examined the self-reported consequences of attending cancer support groups and surveyed what these groups provided to cancer patients that other supportive relationships did not. The most common benefit noted by the support group attendees was a feeling of increased empowerment and agency. Patients felt that support group attendance and connecting with other patients with a cancer diagnosis gave them a stronger sense of control, a better understanding of living with cancer, and positive interactions with others (especially concerning the medical profession). The support groups relieved the family and friends of the patients of the burden of care and provided a safe space for expression for those connected to the patients in question. In doing so, this strengthened these relationships and reduced interpersonal stress, as reported by the participants in this study [100].

\section{Future Recommendations}

Cancer treatment in patients with psychotic mental disorders is very complex and needs more attention. Dispelling the stigma attached to mental illnesses and enrolling more mentally ill patients in clinical trials is the first step to improve their prognosis. In their study of cancer in the SMI, Irwin et al (2019) recommended a bridge intervention, which is a more person-centered approach of collaborative efforts of patients, psychiatrists, social workers, oncologists, and family members to help them better navigate the multiple facets of cancer care [101]. Evidence-based practice with newer and better psychotherapeutic and psychopharmacological interventions with fewer side effects is needed to provide these patients with a better quality of life. In order to ensure equity in cancer care, we encourage large-scale, well-designed studies to identify the risk factors and associations between mental illness and cancer.

\section{Conclusions}

To summarize, the diagnosis and treatment of cancer in patients with pre-existing SMIs, such as bipolar disorder and schizophrenia, can be challenging. Although the incidence of cancer is the same as in the general population, mortality rates have been shown to be higher. There are many risk factors potentiating cancer in patients with SMI, including cigarette smoking, use of antipsychotic medication, poor self-care, lack of timely screening, and poor access to medical care. There are significant barriers that play a role in treating these patients. Due to the apparent stigma associated with mental health and acuity related to cancer symptoms, physicians may not give them the same attention as other patients, which can negatively impact timely cancer screening and diagnosis and may even undermine the cancer symptoms, causing them to go undetected until the cancer metastasizes. Patients with schizophrenia and bipolar disorder may exhibit poor cognitive functioning, and difficulty with comprehension of the seriousness of their cancer diagnosis. These patients may have financial and social constraints and may be unable to adhere to treatment and follow-up care. Oncologists may be hesitant 
in enrolling these patients in clinical trials or recommend intensive treatment due to their noncompliance. Cancer diagnosis and treatment are stressors that may cause new-onset depression or anxiety and exacerbate pre-existing mental illnesses. All these barriers discussed can cause disparity in the diagnosis and treatment of cancer in patients with SMI; therefore, a more targeted approach to cancer care is needed in this challenging population.

\section{Acknowledgments}

The project was approved by the Institutional Review Board (IRB) of the University of Missouri Kansas City. This work would not have been possible without the academic support of UMKC, Department of Psychiatry and Center for Behavioral Medicine. We are grateful to all of those with whom we have had the pleasure to work during this and other related projects. We thank our colleagues who provided insight and expertise that greatly assisted the research, although they may not agree with all of the interpretations/conclusions of this paper. We thank our colleagues and attendings for comments that greatly improved the manuscript.

\section{Financial Disclosure}

No funding to declare. We have no financial activities to disclose. We have no financial relationships associated with the submitting author and all co-authors.

\section{Conflict of Interest}

All authors have no conflict of interest to report. We did not receive any payment or services from a third party for any aspect of the submitted work. We do not have any patents, whether planned, pending or issued, that could be considered broadly relevant to this work.

\section{Author Contributions}

All authors of this research paper have directly participated in the acquisition, analysis, or interpretation of data for the work. NR, KD assisted with data analysis and interpretation of clinical data from the EMR. NR, KD, AD, SD, RV, SP, and SS supplied the acquisition and interpretation of data from the literature, drafting of manuscript. VB provided the conception and design of the review, revised the manuscript critically for important intellectual content prior and gave final approval of the version to be submitted.

\section{Data Availability}

Data are available within the article and in a public repository that issues datasets with DOIs.

\section{References}

1. Chou FH, Tsai KY, Wu HC, Shen SP. Cancer in patients with schizophrenia: What is the next step? Psychiatry Clin Neurosci. 2016;70(11):473-488.

2. Charlson FJ, Ferrari AJ, Santomauro DF, Diminic S, Stockings E, Scott JG, McGrath JJ, et al. Global Epidemiology and Burden of Schizophrenia: Findings From the Global Burden of Disease Study 2016. Schizophr Bull. 2018;44(6):1195-1203.

3. NIMH. Bipolar disorder. Mental Health Information. 2021. https://www.nimh.nih.gov/health/statistics/bipolardisorder.

4. Catts VS, Catts SV, O'Toole BI, Frost AD. Cancer incidence in patients with schizophrenia and their first-degree relatives - a meta-analysis. Acta Psychiatr Scand. 2008;117(5):323-336.

5. Howard LM, Barley EA, Davies E, Rigg A, Lempp H, Rose D, Taylor D, et al. Cancer diagnosis in people with severe mental illness: practical and ethical issues. Lancet Oncol. 2010;11(8):797-804.

6. Agay N, Flaks-Manov N, Nitzan U, Hoshen MB, Levkovitz Y, Munitz H. Cancer prevalence in Israeli men and women with schizophrenia. Psychiatry Res. 2017;258:262-267.

7. Cohen M, Dembling B, Schorling J. The association between schizophrenia and cancer: a population-based mortality study. Schizophr Res. 2002;57(2-3):139-146.

8. Yovel G, Sirota P, Mazeh D, Shakhar G, Rosenne E, BenEliyahu S. Higher natural killer cell activity in schizophrenic patients: the impact of serum factors, medication, and smoking. Brain Behav Immun. 2000;14(3):153-169.

9. Catts VS, Catts SV. Apoptosis and schizophrenia: is the tumour suppressor gene, p53, a candidate susceptibility gene? Schizophr Res. 2000;41(3):405-415.

10. Ciernikova S, Mego M, Chovanec M. Exploring the potential role of the gut microbiome in chemotherapy-induced neurocognitive disorders and cardiovascular toxicity. Cancers (Basel). 2021;13(4):782.

11. Watkins CC, Sawa A, Pomper MG. Glia and immune cell signaling in bipolar disorder: insights from neuropharmacology and molecular imaging to clinical application. Transl Psychiatry. 2014;4:e350.

12. Barichello T, Simoes LR, Quevedo J, Zhang XY. Microglial activation and psychotic disorders: evidence from pre-clinical and clinical studies. Curr Top Behav Neurosci. 2020;44:161-205.

13. Momtazmanesh S, Zare-Shahabadi A, Rezaei N. Cytokine Alterations in Schizophrenia: An Updated Review. Front Psychiatry. 2019;10:892.

14. Alda M. Lithium in the treatment of bipolar disorder: pharmacology and pharmacogenetics. Mol Psychiatry. 2015;20(6):661-670.

15. Peuskens J, Pani L, Detraux J, De Hert M. The effects of novel and newly approved antipsychotics on serum prolactin levels: a comprehensive review. CNS Drugs. 2014;28(5):421-453.

16. Griswold KS, Del Regno PA, Berger RC. Recognition 
and differential diagnosis of psychosis in primary care. Am Fam Physician. 2015;91(12):856-863.

17. Garg H, Prakash S, Deb KS, Chadda RK. Secondary mania following cancer chemotherapy with capecitabine. BMJ Case Rep. 2018;2018:bcr2017220995.

18. Barley EA, Borschmann RD, Walters P, Tylee A. Interventions to encourage uptake of cancer screening for people with severe mental illness. Cochrane Database Syst Rev. 2016;9:CD009641.

19. M DEH, Correll CU, Bobes J, Cetkovich-Bakmas M, Cohen D, Asai I, Detraux J, et al. Physical illness in patients with severe mental disorders. I. Prevalence, impact of medications and disparities in health care. World Psychiatry. 2011;10(1):52-77.

20. Hwong A, Wang K, Bent S, Mangurian C. Breast Cancer Screening in Women With Schizophrenia: A Systematic Review and Meta-Analysis. Psychiatr Serv. 2020;71(3):263-268.

21. Obuchi T, Okabayashi K, Imakiire T, Yoneda S, Iwasaki A. Outcomes of surgery in lung cancer patients with schizophrenia. Surg Today. 2014;44(5):855-858.

22. Irwin KE, Henderson DC, Knight HP, Pirl WF. Cancer care for individuals with schizophrenia. Cancer. 2014;120(3):323-334.

23. Weinstein LC, Stefancic A, Cunningham AT, Hurley KE, Cabassa LJ, Wender RC. Cancer screening, prevention, and treatment in people with mental illness. CA Cancer J Clin. 2016;66(2):134-151.

24. Miovic M, Block S. Psychiatric disorders in advanced cancer. Cancer. 2007;110(8):1665-1676.

25. Lawlor PG, Gagnon B, Mancini IL, Pereira JL, Hanson J, Suarez-Almazor ME, Bruera ED. Occurrence, causes, and outcome of delirium in patients with advanced cancer: a prospective study. Arch Intern Med. 2000;160(6):786794.

26. Caraceni A, Nanni O, Maltoni M, Piva L, Indelli M, Arnoldi E, Monti M, et al. Impact of delirium on the short term prognosis of advanced cancer patients. Italian Multicenter Study Group on Palliative Care. Cancer. 2000;89(5):1145-1149.

27. Wikman A, Ljung R, Johar A, Hellstadius Y, Lagergren J, Lagergren P. Psychiatric morbidity and survival after surgery for esophageal cancer: a population-based cohort study. J Clin Oncol. 2015;33(5):448-454.

28. Bernad DM, Dal Pra A, Baule C, Frey BN, Faria S. Newonset psychosis following androgen deprivation therapy for prostate cancer. Can J Urol. 2013;20(4):6868-6870.

29. Campbell BA, Panicker J. New onset psychosis in an adolescent during treatment of testicular germ cell tumor. J Pediatr Hematol Oncol. 2011;33(3):e125-126.

30. Inagaki T, Yasukawa R, Okazaki S, Yasuda H, Kawamukai T, Utani E, Hayashida M, et al. Factors disturbing treatment for cancer in patients with schizophrenia. Psychiatry Clin Neurosci. 2006;60(3):327-331.

31. Kohler O, Sylvia LG, Bowden CL, Calabrese JR, Thase M, Shelton RC, McInnis M, et al. White blood cell count correlates with mood symptom severity and specific mood symptoms in bipolar disorder. Aust N Z J Psychiatry. 2017;51(4):355-365.
32. Nagamine T. Abnormal laboratory values during the acute and recovery phases in schizophrenic patients: a retrospective study. Neuropsychiatr Dis Treat. 2010;6:281288.

33. Falcone T, Carlton E, Lee C, Janigro M, Fazio V, Forcen FE, Franco K, et al. Does systemic inflammation play a role in pediatric psychosis? Clin Schizophr Relat Psychoses. 2015;9(2):65-78B.

34. Druss BG, Rosenheck RA, Desai MM, Perlin JB. Quality of preventive medical care for patients with mental disorders. Med Care. 2002;40(2):129-136.

35. Seeman MV. Schizophrenia and cancer: low incidence, high mortality. Res J Oncol. 2017;1. iMedPub. http:// www.imedpub.com/research-journal-oncology/.

36. Haskins CB, McDowell BD, Carnahan R, Fiedorowicz J, Wallace RB, Smith BJ, Chrischilles EA. Prevalence of preexisting mental illness in SEER-Medicare breast cancer patients. J Clin Oncol. 2018;36(15_suppl):e13564e13564.

37. Kahan NR, Silverman B, Liphshitz I, Waitman DA, BenZion I, Ponizovsky AM, Weizman A, et al. No apparent association between bipolar disorder and cancer in a large epidemiological study of outpatients in a managed care population. Int Clin Psychopharmacol. 2018;33(2):73-78.

38. Lin GM, Chen YJ, Kuo DJ, Jaiteh LE, Wu YC, Lo TS, Li YH. Cancer incidence in patients with schizophrenia or bipolar disorder: a nationwide population-based study in Taiwan, 1997-2009. Schizophr Bull. 2013;39(2):407416.

39. Zhuo C, Zhuang H, Gao X, Triplett PT. Lung cancer incidence in patients with schizophrenia: meta-analysis. Br J Psychiatry. 2019;215(6):704-711.

40. Laursen TM, Munk-Olsen T, Gasse C. Chronic somatic comorbidity and excess mortality due to natural causes in persons with schizophrenia or bipolar affective disorder. PLoS One. 2011;6(9):e24597.

41. Kisely S, Smith M, Lawrence D, Cox M, Campbell LA, Maaten S. Inequitable access for mentally ill patients to some medically necessary procedures. CMAJ. 2007;176(6):779-784.

42. Atik L, Konuk N, Akay O, Ozturk D, Erdogan A. Pain perception in patients with bipolar disorder and schizophrenia. Acta Neuropsychiatr. 2007;19(5):284-290.

43. Sullivan G, Han X, Moore S, Kotrla K. Disparities in hospitalization for diabetes among persons with and without co-occurring mental disorders. Psychiatr Serv. 2006;57(8):1126-1131.

44. Druss BG, Bradford WD, Rosenheck RA, Radford MJ, Krumholz HM. Quality of medical care and excess mortality in older patients with mental disorders. Arch Gen Psychiatry. 2001;58(6):565-572.

45. Fleming ST, Pursley HG, Newman B, Pavlov D, Chen K. Comorbidity as a predictor of stage of illness for patients with breast cancer. Med Care. 2005;43(2):132-140.

46. Johnston AN, Bu W, Hein S, Garcia S, Camacho L, Xue L, Qin L, et al. Hyperprolactinemia-inducing antipsychotics increase breast cancer risk by activating JAK-STAT5 in precancerous lesions. Breast Cancer Res. 2018;20(1):42.

47. George A, Sturgeon SR, Hankinson SE, Shadyab AH, 
Wallace RB, Reeves KW. Psychotropic medication use and postmenopausal breast cancer risk. Cancer Epidemiol Biomarkers Prev. 2020;29(1):254-256.

48. Badran A, Tul-Wahab A, Zafar H, Mohammad N, Imad R, Ashfaq Khan M, Baydoun E, et al. Antipsychotics drug aripiprazole as a lead against breast cancer cell line (MCF-7) in vitro. PLoS One. 2020;15(8):e0235676.

49. Shaw V, Srivastava S, Srivastava SK. Repurposing antipsychotics of the diphenylbutylpiperidine class for cancer therapy. Semin Cancer Biol. 2021;68:75-83.

50. Hendouei N, Saghafi F, Shadfar F, Hosseinimehr SJ. Molecular mechanisms of anti-psychotic drugs for improvement of cancer treatment. Eur J Pharmacol. 2019;856:172402.

51. Sakamoto R, Koyama A. Effective therapy against severe anxiety caused by cancer: a case report and review of the literature. Cureus. 2020;12(6):e8414.

52. Shinno H, Matsuoka T, Yamamoto O, Noma Y, Hikasa S, Takebayashi M, Horiguchi J. Successful treatment with quetiapine for delirium in terminally ill cancer patients. Psychogeriatrics, 2007;7:64-68.

53. Verwimp-Hoeks MPA, van Herpen CML, Burger DM. Aprepitant quetiapine: a clinically significant drug interaction in a patient treated for head and neck cancer. Ann Oncol. 2012;23(3):801-802.

54. Sanomachi T, Suzuki S, Kuramoto K, Takeda H, Sakaki H, Togashi K, Seino S, et al. Olanzapine, an Atypical Antipsychotic, Inhibits Survivin Expression and Sensitizes Cancer Cells to Chemotherapeutic Agents. Anticancer Res. 2017;37(11):6177-6188.

55. Bitter I, Dossenbach MR, Brook S, Feldman PD, Metcalfe S, Gagiano CA, Furedi J, et al. Olanzapine versus clozapine in treatment-resistant or treatment-intolerant schizophrenia. Prog Neuropsychopharmacol Biol Psychiatry. 2004;28(1):173-180.

56. Breitbart W, Tremblay A, Gibson C. An open trial of olanzapine for the treatment of delirium in hospitalized cancer patients. Psychosomatics. 2002;43(3):175-182.

57. Kishi Y, Kato M, Okuyama T, Thurber S. Treatment of delirium with risperidone in cancer patients. Psychiatry Clin Neurosci. 2012;66(5):411-417.

58. Reutfors J, Wingard L, Brandt L, Wang Y, Qiu H, Kieler H, Bahmanyar S. Risk of breast cancer in risperidone users: A nationwide cohort study. Schizophr Res. 2017;182:98103.

59. McIver B, Walsh D, Nelson K. The use of chlorpromazine for symptom control in dying cancer patients. J Pain Symptom Manage. 1994;9(5):341-345.

60. Kamgar-Dayhoff P, Brelidze TI. Multifaceted effect of chlorpromazine in cancer: implications for cancer treatment. Oncotarget. 2021;12(14):1406-1426.

61. Csatary LK. Chlorpromazines and cancer. Lancet. 1972;2(7772):338-339.

62. Radha Krishna LK, Poulose VJ, Goh C. The use of midazolam and haloperidol in cancer patients at the end of life. Singapore Med J. 2012;53(1):62-66.

63. Hui D, Frisbee-Hume S, Wilson A, Dibaj SS, Nguyen T, De La Cruz M, Walker P, et al. Effect of lorazepam with haloperidol vs haloperidol alone on agitated delirium in patients with advanced cancer receiving palliative care: a randomized clinical trial. JAMA. 2017;318(11):10471056.

64. Wang EH, Mabasa VH, Loh GW, Ensom MH. Haloperidol dosing strategies in the treatment of delirium in the critically ill. Neurocrit Care. 2012;16(1):170-183.

65. Riddle M, Markle C, Combs Heidi, Croicu C, et al. Managing schizophrenia in a patient with cancer: A fine balance. Current Psychiatry. 2017;16:42-47.

66. Schmiedl S, Peters D, Schmalz O, Mielke A, Rossmanith T, Diop S, Piefke M, et al. Loxapine for treatment of patients with refractory, chemotherapy-induced neuropathic pain: a prematurely terminated pilot study showing efficacy but limited tolerability. Front Pharmacol. 2019;10:838.

67. Rahman T, Clevenger CV, Kaklamani V, Lauriello J, Campbell A, Malwitz K, Kirkland RS. Antipsychotic treatment in breast cancer patients. Am J Psychiatry. 2014;171(6):616-621.

68. Deodhar JK, Prabhash K, Agarwal JP, Chaturvedi P. Clozapine and cancer treatment: Adding to the experience and evidence. Indian J Psychiatry. 2014;56(2):191-193.

69. Pakhre A, Krishnan A, Pattanayak RD, Khandelwal SK. Use of clozapine alongside chemotherapy in a treatmentresistant bipolar disorder patient with ovarian carcinoma: A case report and brief review. Indian J Psychiatry. 2016;58(4):462-466.

70. Thomson K, Henry B. Oncology clinical challenges: caring for patients with preexisting psychiatric illness. Clin J Oncol Nurs. 2012;16(5):471-480.

71. Yoon HJ, Park KM, Choi WJ, Choi SH, Park JY, Kim JJ, Seok JH. Efficacy and safety of haloperidol versus atypical antipsychotic medications in the treatment of delirium. BMC Psychiatry. 2013;13:240.

72. Grassi L, Caruso R, Hammelef K, Nanni MG, Riba M. Efficacy and safety of pharmacotherapy in cancer-related psychiatric disorders across the trajectory of cancer care: a review. Int Rev Psychiatry. 2014;26(1):44-62.

73. Howell S, Yarovova E, Khwanda A, Rosen SD. Cardiovascular effects of psychotic illnesses and antipsychotic therapy. Heart. 2019;105(24):1852-1859.

74. Zhu J, Hou W, Xu Y, Ji F, Wang G, Chen C, Lin C, et al. Antipsychotic drugs and sudden cardiac death: A literature review of the challenges in the prediction, management, and future steps. Psychiatry Res. 2019;281:112598.

75. Kilian JG, Kerr K, Lawrence C, Celermajer DS. Myocarditis and cardiomyopathy associated with clozapine. Lancet. 1999;354(9193):1841-1845.

76. Grajales D, Ferreira V, Valverde AM. Second-generation antipsychotics and dysregulation of glucose metabolism: beyond weight gain. Cells. 2019;8(11):1336.

77. Dietrich-Muszalska A, Wachowicz B. Platelet haemostatic function in psychiatric disorders: Effects of antidepressants and antipsychotic drugs. World J Biol Psychiatry. 2017;18(8):564-574.

78. Swarm RA, Paice JA, Anghelescu DL, Are M, Bruce JY, Buga S, Chwistek M, et al. Adult Cancer Pain, Version 3.2019, NCCN Clinical Practice Guidelines in Oncology. J Natl Compr Canc Netw. 2019;17(8):977-1007.

79. Khojainova N, Santiago-Palma J, Kornick C, Breitbart W, 
Gonzales GR. Olanzapine in the management of cancer pain. J Pain Symptom Manage. 2002;23(4):346-350.

80. Heo MH, Kim JY, Hwang I, Ha E, Park KU. Analgesic effect of quetiapine in a mouse model of cancer-induced bone pain. Korean J Intern Med. 2017;32(6):1069-1074.

81. Ha JH, Hwang DY, Yu J, Park DH, Ryu SH. Onset of Manic Episode during Chemotherapy with 5-Fluorouracil. Psychiatry Investig. 2011;8(1):71-73.

82. Hariram J, Jegan Y. Contribution of methotrexate in precipitation of manic episode in bipolar affective disorder explored: a case report. Ther Adv Psychopharmacol. 2013;3(4):251-254.

83. Barlinn K, Lehrach H, Siepmann T, Braeuer D, Buntrock U, Sassim N. Temporary loss of moral behavior in a patient undergoing chemotherapy with cisplatin - breaking bad. BMC Psychiatry. 2015;15:9.

84. DeKay JW, Matuszak J. A 68-year-old woman with lung cancer and late-onset bipolar disorder. Psychiatric Annals. 2011;41(5):261-263.

85. Matsunaga M, Onishi H, Ishida M, Miwa K, Araki K, Kaneta T, Sunakawa Y, et al. Hypomanic episode during recurrent gastric cancer treatment: report of a rare case and literature review. Jpn J Clin Oncol. 2012;42(10):961-964.

86. Warren KN, Katakam J, Espiridion ED. Acute-onset mania in a patient with non-small cell lung cancer. Cureus. 2019;11(8):e5436.

87. Olesya Pavlova, Wolfram Weinrebe. Chemotherapy-induced fulminant, severe hyperactive delirium in a patient with a new diagnosed multiple myeloma. Archives of Clinical and Medical Case Reports. 2020;4:312-328.

88. Sami MB, Yousaf F, Fialho A. Severe agitation in depression precipitated by dasatinib. BMJ Case Rep. 2014;2014:bcr2014204166.

89. Quek R, Morgan JA, George S, Butrynski JE, Polson K, Meyer F, Demetri GD, et al. Small molecule tyrosine kinase inhibitor and depression. J Clin Oncol. 2009;27(2):312-313.

90. Li B, Thrasher JB, Terranova P. Glycogen synthase kinase-3: a potential preventive target for prostate cancer management. Urol Oncol. 2015;33(11):456-463.
91. Thakur S, Tobey A, Klubo-Gwiezdzinska J. The role of lithium in management of endocrine tumors-a comprehensive review. Front Oncol. 2019;9:1092.

92. Elmaci I, Altinoz MA. A Metabolic Inhibitory Cocktail for Grave Cancers: Metformin, Pioglitazone and Lithium Combination in Treatment of Pancreatic Cancer and Glioblastoma Multiforme. Biochem Genet. 2016;54(5):573618.

93. Slingerland M, Guchelaar HJ, Gelderblom H. Histone deacetylase inhibitors: an overview of the clinical studies in solid tumors. Anticancer Drugs. 2014;25(2):140-149.

94. Koong SS, Reynolds JC, Movius EG, Keenan AM, Ain KB, Lakshmanan MC, Robbins J. Lithium as a potential adjuvant to $131 \mathrm{I}$ therapy of metastatic, well differentiated thyroid carcinoma. J Clin Endocrinol Metab. 1999;84(3):912-916.

95. Mehta RD, Roth AJ. Psychiatric considerations in the oncology setting. CA Cancer J Clin. 2015;65(4):300-314.

96. Radbruch L, Strasser F, Elsner F, Goncalves JF, Loge J, Kaasa S, Nauck F, et al. Fatigue in palliative care patients — an EAPC approach. Palliat Med. 2008;22(1):13-32.

97. Okuyama T, Akechi T, Mackenzie L, Furukawa TA. Psychotherapy for depression among advanced, incurable cancer patients: A systematic review and meta-analysis. Cancer Treat Rev. 2017;56:16-27.

98. Lang-Rollin I, Berberich G. Psycho-oncology. Dialogues Clin Neurosci. 2018;20(1):13-22.

99. Arch JJ, Mitchell JL. An Acceptance and Commitment Therapy (ACT) group intervention for cancer survivors experiencing anxiety at re-entry. Psychooncology. 2016;25(5):610-615.

100. Ussher J, Kirsten L, Butow P, Sandoval M. What do cancer support groups provide which other supportive relationships do not? The experience of peer support groups for people with cancer. Soc Sci Med. 2006;62(10):25652576.

101. Irwin KE, Park ER, Fields LE, Corveleyn AE, Greer JA, Perez GK, Callaway CA, et al. Bridge: person-centered collaborative care for patients with serious mental illness and cancer. Oncologist. 2019;24(7):901-910. 